\title{
Effect of a Dementia Diagnosis on Survival of Older Patients After a Diagnosis of Breast, Colon, or Prostate Cancer:
}

\author{
Implications for Cancer Care
}

\author{
Mukaila A. Raji, MD, MSc, Yong-Fang Kuo, PhD, Jean L. Freeman, PhD, and James S. \\ Goodwin, MD \\ Department of Internal Medicine, Sealy Center on Aging, University of Texas Medical Branch, \\ Galveston
}

\begin{abstract}
Background-Preexisting dementia affects cancer care. Knowledge of how dementia affects survival after a cancer diagnosis may help guide cancer care decisions. We therefore examined the associations between preexisting diagnoses of dementia and survival from breast, colon, and prostate cancer.

Methods-We conducted a retrospective cohort study of 106061 patients aged 68 years or older diagnosed as having breast, colon, or prostate cancer, using data from the linked Surveillance, Epidemiology and End Results- Medicare database. We assessed the risks of mortality from cancer and noncancer causes, stratified by presence or absence of preexisting dementia diagnoses. Cox proportional hazards regression was used to adjust for confounding variables.
\end{abstract}

\begin{abstract}
Results-Seven percent of our sample had preexisting dementia diagnoses. Survival after a cancer diagnosis was markedly worse in demented than in nondemented patients. Most of the excess deaths came from noncancer causes; $33.3 \%$ of those with a dementia diagnosis died within 6 months of a cancer diagnosis, compared with $8.5 \%$ of patients without dementia. Less than $17.0 \%$ of the excess mortality in patients with dementia who had breast or colon cancer was explained by a more advanced cancer stage at diagnosis. None of the excess deaths in prostate cancer was explained by stage at diagnosis. For all 3 cancers, the presence of preexisting dementia diagnoses attenuated the relationship between stage at diagnosis and survival.
\end{abstract}

\footnotetext{
(C2008 American Medical Association. All rights reserved.

Correspondence: Mukaila A. Raji, MD, MSc, Memory Loss Clinic, Division of Geriatrics, Department of Internal Medicine, Sealy Center on Aging, University of Texas Medical Branch, 301 University Blvd, Rte 0460, Galveston, TX 77555-0460 (muraji@utmb.edu).

Author Contributions: All authors had full access to all of the data in the study and take responsibility for the integrity of the data and the accuracy of the data analysis. Study concept and design: Raji and Goodwin. Acquisition of data: Kuo and Freeman. Analysis and interpretation of data: Raji, Kuo, Freeman, and Goodwin. Drafting of the manuscript: Raji. Critical revision of the manuscript for important intellectual content: Raji, Kuo, Freeman, and Goodwin. Statistical analysis: Kuo. Obtained funding: Freeman and Goodwin Administrative, technical, and material support: Raji, Kuo, and Freeman. Study supervision: Raji and Goodwin.
}

Financial Disclosure: None reported.

Role of Sponsor: The sponsors had no role in the design, methods, subject recruitment, data collection, analysis, or preparation of the manuscript.

Additional Contributions: We acknowledge the efforts of the Applied Research Program, National Cancer Institute; the Office of Research, Development, and Information, Centers for Medicare and Medicaid Services; Information Management Services Inc; and the SEER Program tumor registries in the creation of the SEER-Medicare database.

Publisher's Disclaimer: Disclaimer: This study used the linked SEER-Medicare database. The interpretation and reporting of these data are the sole responsibility of the authors. 
Conclusions-Preexisting dementia diagnoses were associated with high mortality, mostly from noncancer causes. The effect of cancer stage at diagnosis on mortality was significantly reduced in older patients with pre-cancer diagnoses of dementia.

The Incidence of both Dementia and cancer increases with age. For those aged 65 years and older, the prevalence of dementia is about 1 in $10 .{ }^{1}$ Approximately 4.5 million Americans have Alzheimer dementia, and the number is projected to triple by the year 2050. ${ }^{1,2}$

Similarly, for those aged 60 to 79 years, the probability of developing invasive cancer is 1 in 3 for men and 1 in 4 for women. ${ }^{3}$ Thus, the probability of co-occurrence of both dementia and cancer in the same patient rises with increasing age.

Cognitive impairment may interfere with the diagnosis and treatment of older patients with cancer. Two recent stud-ies ${ }^{4,5}$ have reported an association of preexisting dementia with later-stage cancer at diagnosis. In their analysis of approximately 17500 older patients with invasive colon cancer, Gupta and Lamont ${ }^{4}$ found that a precancer dementia diagnosis was associated with lower odds of undergoing diagnostic tissue biopsy and higher odds of having unstaged cancer. In another study ${ }^{5}$ of 50460 older patients with breast cancer, a diagnosis of dementia was independently associated with late-stage breast cancer at diagnosis. There is, however, little information on how dementia affects survival after a cancer diagnosis.

Dementia increases the risk of noncancer deaths in patients with can-cer. ${ }^{6}$ Pneumonia is the most common cause of death in severe dementia, while heart disease and stroke are common causes of death in mild to moderate dementia. ${ }^{7}$

Knowing how comorbid dementia affects deaths from cancer and from noncancer causes may help guide cancer care decisions. Such knowledge may also help answer important clinical questions. Is screening for cancer appropriate in patients with dementia diagnoses? What is the appropriate level of care (eg, palliative vs aggressive treatment) for cancer in the context of preexisting dementia diagnoses? The present study examined the effect of a preexisting diagnosis of dementia on deaths from cancer and other causes in older adults diagnosed as having cancer of the breast, colon, or prostate. These sites were chosen because commonly used screening tests exist for each of them. We identified patients with dementia diagnoses through Medicare claims linked to the National Cancer Institute's Surveillance, Epidemiology and End Results (SEER) tumor registry data. ${ }^{5,6,8,9}$ We hypothesized that the presence of dementia diagnoses would be associated with increased mortality from cancer and noncancer causes in older adults with these cancers. We further hypothesized that the stage of the cancer at diagnosis would have less of an effect on survival in patients with dementia than on those without dementia.

\section{Methods}

The present study was reviewed and approved by the institutional review board of the University of Texas Medical Branch.

\section{Data Sources}

We used SEER-Medicare linked data for this study. ${ }^{8} 9$ The SEER program supports population-based cancer registries covering $14 \%$ of the US population. Ninety-three percent of those with cancers at 65 years or older were matched with their Medicare enrollment records. The SEER-Medicare database includes patient-level demographic and cancerspecific variables: age, ethnicity, type of cancer, month and year of cancer diagnosis, and extent of cancer (eg, stage, tumor size/ extension, and histologic grade). It also includes selected socioeconomic characteristics of the census tract where the patient resides at the time of diagnosis. 


\section{Identification of Patients with Cancer}

Our study population was selected from SEER data in the Patient Entitlement and Diagnosis File. It included all Medicare beneficiaries diagnosed as having cancer from January 1994 through December 1999. The population comprised 2 groups, defined on the basis of comorbid dementia with claims data. The group with comorbid dementia included Medicare beneficiaries who received a diagnosis of dementia and related disorders between January 1991 and December 1999, within the 3 years before their cancer diagnosis. The comparison group was participants with no known diagnosis of dementia. We excluded all individuals younger than 68 years at the time of diagnosis, those who did not have full coverage of Medicare Parts A and B, and those who were enrolled in a health maintenance organization at any time during the period from 3 years before the diagnosis of cancer to December 31, 2001, or death. Our sample thus comprised 106061 patients diagnosed as having breast, colon, or prostate cancer.

\section{Identification of Patients with a Preexisting Diagnosis of Dementia}

We used the method described by Taylor et a ${ }^{10}$ to identify all Medicare patients with a preexisting dementia diagnosis using diagnosis codes as recorded in the Medicare claims data files during the 3-year period before cancer diagnosis. Taylor and colleagues linked up to 5 years of Medicare claims data to clinical files of more than 400 patients known to have a clinical diagnosis of dementia. The claims data files included the physician outpatient and the physician supplier claim files. Any dementia diagnosis at any visit and at any position of the listed diagnoses by the providers was used. With 3 years of data from physician supplier and physician outpatient claim files, the investigators were able to correctly identify $87 \%$ of their patients with dementia by using general International Classification of Diseases, Ninth Revision, Clinical Modification(ICD-9-CD) dementia codes as listed. Adding hospital files or using 5 years of claims data had little effect on the dementia identification rate. The ICD-9-CM codes at any visit and in any position from inpatient, hospital outpatient, and physician claims were used to identify patients with diagnoses of dementia. The ICD-9-CM codes used to define Alzheimer disease and related dementias were 331.0 to 331.2, 331.7, $290.0,290.1,290.10$ to $290.13,290.20,290.21,290.3,290.40$ to $290.43,294.0,294.1$, 294.8 , and 797.

\section{Measures}

Patient characteristics obtained from SEER included age, sex, ethnicity, marital status, type of cancer, and date of cancer diagnosis. Medicare data provide information on level of comorbidity (derived from modification by Klabunde et al ${ }^{11}$ of the Charlson Comorbidity Index). The Charlson Comorbidity Index comprises 19 conditions identified from inpatient hospital claims (Medicare Part A). The Klabunde et al-modified Charlson Comorbidity Index includes diagnostic and procedure information from physician outpatient claims (Medicare Part B). For our analysis, dementia was not included in the Klabunde et al modification of the Charlson Comorbidity Index. Census tract characteristics include the percentage of adults with less than 12 years of education and the percentage of those living below the poverty line.

Tumor characteristics obtained from SEER included American Joint Committee on Cancer stage for breast and colon cancers and clinical stage (using the SEER Extent-of-Disease classification system) for prostate cancer, histologic grade (well, moderate, poorly differentiated, or undetermined), and, in the case of breast cancer, hormone receptor status. 


\section{Statistical Analysis}

The proportion of patients with a preexisting dementia diagnosis across strata of patient characteristics and the distribution of stage at diagnosis between patients with and without dementia for each cancer were calculated. The Kaplan-Meier method was used to generate survival curves for patients with and without a preexisting dementia diagnosis. Two Cox proportional hazard models were built to test the association between a preexisting dementia diagnosis and cancer-specific mortality and overall mortality, with and without additional adjustment for cancer stage, among each type of cancer. The mediation effect of cancer stage on the association between a preexisting dementia diagnosis and mortality was estimated by the percentage change of additional risk from dementia between the two Cox models. ${ }^{12}$ In these survival analyses, patient data were censored on December 31, 2001. The interaction between a preexisting dementia diagnosis and cancer stage on the relative risk of mortality (moderation effect) was evaluated with a Cox model for each type of cancer. The cancer and noncancer excess mortality associated with dementia was estimated from logistic regression models adjusted for confounding variables. In these logistic regression models, the outcomes of cancer death and noncancer death were modeled separately. For the calculation of excess mortality over 5 years, only patients diagnosed from January 1994 through December 1996 with 5 years of follow-up were included. All analyses were performed with the use of SAS version 9.1 (SAS Institute Inc, Cary, North Carolina).

\section{Results}

Our sample was composed of 31935 patients with breast cancer, 26891 with colon cancer, and 47235 with prostate cancer (Table 1). The prevalence of precancer diagnoses of dementia varied by cancer: $7.4 \%$ in patients with breast cancer, $10.0 \%$ for colon cancer, and $5.1 \%$ for prostate cancer. All differences in prevalences of dementia by demographic characteristics were significant at $P<.001$. Preexisting diagnoses of dementia were more prevalent in patients who were older, black, female, unmarried, less educated, and living in areas with a high percentage of poverty (Table 1). Preexisting diagnoses of dementia also increased with number of comorbidities.

\section{Effect of a Dementia Diagnosis on Cancer Stage at Diagnosis}

Patients with cancer who had preexisting diagnoses of dementia were significantly less likely to be diagnosed at an early stage of cancer (Table 2). This likelihood varied by cancer site, with the highest effect in breast cancer: $28.0 \%$ of patients with breast cancer with dementia were diagnosed at stage 1 compared with $50.7 \%$ of those without dementia. Patients with dementia diagnoses were also significantly more likely to be diagnosed at an unknown stage of cancer. For example, $22.5 \%$ to $22.7 \%$ of patients with dementia (vs $6.4 \%$ to $7.5 \%$ of patients without dementia) were diagnosed at an unknown stage of colon or breast cancer, respectively.

\section{Effect of a Dementia Diagnosis on Survival}

The Figure presents survival curves for patients with breast, colon, and prostate cancer, stratified by whether they carried a preexisting diagnosis of dementia. Approximately $33.3 \%$ of patients with dementia vs $8.5 \%$ of those without it died within 6 months of a cancer diagnosis. The effect of dementia on death within 6 months differed by cancer site: breast, $24.9 \%$ vs $5.1 \%$; colon, $44.9 \%$ vs $20.2 \%$; and prostate, $28.5 \%$ vs $4.3 \%$.

Table 3 presents mortality at 1 and 5 years after a cancer diagnosis for patients with and without a preexisting diagnosis of dementia. These analyses were adjusted for age, other comorbidities, and other demographic factors. The analyses separately assess deaths from cancer and deaths from all other causes. For each of the 3 cancers, dementia was associated 
with a greater absolute increase in deaths from noncancer causes than from cancer causes. For example, the adjusted percentage dead from noncancer causes within 5 years of a breast cancer diagnosis was $31.8 \%$ in patients with dementia and $16.2 \%$ in patients without dementia, a $15.6 \%$ absolute difference. By contrast, the absolute difference in 5-year mortality from cancer between patients with breast cancer with and without dementia was $4.8 \%$. For prostate cancer, the absolute difference between demented and non-demented patients was $12.7 \%$ for noncancer deaths vs $2.8 \%$ for cancer deaths, and for colon cancer the respective values were $12.8 \%$ vs $3.5 \%$. Similar patterns were seen with 1 -year mortality rates (Table 3).

Table 4 presents 3 sets of adjusted hazard rates of mortality: mortality from breast, colon, and prostate cancer; mortality from other causes; and all-cause mortality, associated with a diagnosis of dementia. In model 1, adjustments were made for age, ethnicity, sex (for colon cancer), marital status, SEER region, educational level, income, comorbidity (other than dementia), year of diagnosis, and other prognostic factors. Model 1 shows that a preexisting diagnosis of dementia was associated with increased mortality from all causes, mortality from cancer, and mortality from noncancer causes in all 3 cancers. In further analyses, we stratified patients with a dementia diagnosis into those with Alzheimer dementia, vascular dementia, and other dementias. All categories were associated with substantially decreased survival (data not shown).

Model 2 in Table 4 examines the effect of stage at diagnosis on the relationship of a dementia diagnosis to survival. Looking at cancer-specific mortality, controlling for stage at diagnosis had a modest effect in patients with breast and colon cancer and no effect in patients with prostate cancer. The effect was still present but smaller when looking at allcause mortality. The mediation effect of cancer stage on the association between a diagnosis of dementia and mortality was calculated from Table 4 : $\left(\mathrm{HR}_{\text {model } 1}-\mathrm{HR}_{\text {model } 2}\right) /\left(\mathrm{HR}_{\text {model }} 1\right.$ - 1), where HR indicates hazard ratio. ${ }^{12}$ Controlling for stage at diagnosis explained $16.4 \%$ of the excess in all-cause mortality in breast cancer associated with a dementia diagnosis, and $13.6 \%$ of the excess in all-cause mortality in colon cancer associated with a diagnosis of dementia (both $P<.001$ ).

In Table 4 there were significant interactions between a diagnosis of dementia and cancer stage at diagnosis on cancer-specific and all-cause survival, for each of the 3 cancers $(P<$. 001 for each interaction). Consequently, we examined the effect of stage at diagnosis on survival, after stratifying by whether there was a previous diagnosis of dementia. As shown in Table 5, the relationship between stage at diagnosis and mortality is diminished in patients with a preexisting diagnosis of dementia. This is true for cancer-specific mortality and for all-cause mortality. For example, the hazard of death from any cause associated with a diagnosis of stage 4 vs stage 1 breast cancer is 10.35 in patients without a dementia diagnosis vs 4.03 in patients with a dementia diagnosis.

\section{Comment}

The results of this study can be summarized as follows. First, the presence of a preexisting diagnosis of dementia was associated with decreased survival after a diagnosis of breast, colon, or prostate cancer. Second, a dementia diagnosis was associated with increased mortality from cancer and from noncancer causes. The absolute increase in deaths associated with a dementia diagnosis was considerably larger from noncancer causes of death than from deaths from cancer. Third, a dementia diagnosis was associated with increased odds of being diagnosed at an unknown stage of cancer. Patients diagnosed at an unknown stage in SEER data tend to have survival profiles similar to those diagnosed with stage $4 .{ }^{13}$ Fourth, for colon and breast cancer, the increase in cancer deaths with a dementia diagnosis is partly 
explained by more advanced stage at diagnosis. Diagnosis at a more advanced cancer stage explained $16.4 \%$ of increased mortality associated with a dementia diagnosis in breast cancer and $13.6 \%$ for colorectal cancer.

In the case of prostate cancer, adjusting for stage had no effect on the cancer-specific mortality associated with dementia. As opposed to breast and colorectal cancer, stage at diagnosis in prostate cancer explains little of the variance in all-cause mortality. ${ }^{14}$ Finally, the effect of stage at diagnosis on cancer-specific or all-cause mortality after a cancer diagnosis was substantially reduced in patients with a dementia diagnosis.

Increasing attention has been paid to challenges encountered when screening or treatment guidelines are applied to older patients. ${ }^{15-17}$ One message is that guidelines generated from studying younger populations are not always appropriate in the elderly. Boyd et al ${ }^{15}$ discussed the issues of multiple comorbidities resulting in a burden of multiple recommended evaluations and resultant polypharmacy. Defining appropriateness of care in the context of comorbidities is complex. The complexity arises in part from the difficulty in balancing disease-specific guidelines with health-related quality of life and life expectancy of elderly patients with multiple co-morbidities. ${ }^{16}$ Also, the decreased life expectancy of older persons might result in a reduced theoretical benefit of many screening tests. Gross and colleagues ${ }^{17}$ discussed this in the context of cancer screening, suggesting that an estimate of life expectancy be used when decisions are made about cancer screening in older patients with multiple comorbidities. Our study adds to this discussion by providing information on survival in 1 important subgroup of older individuals: those with a diagnosis of dementia.

The patient with dementia is a challenge to any health care provider. Issues of consent for testing become more difficult. In addition, in patients with moderate to severe dementia, a seemingly minor process such as a blood draw or screening mammogram might be perceived by the patient as an assault, thereby greatly increasing the emotional distress of the procedure for the patient, the care-givers, and the provider. In short, the cost-benefit analysis may be altered by the increase in the emotional costs for the participants. Our results suggest that the benefit of such screening is also altered. Survival after a cancer diagnosis is much worse among older subjects with a dementia diagnosis than in those without a diagnosis of comorbid dementia. Most of the excess mortality was not from the cancer. This suggests that better cancer screening or treatment of those patients would not greatly affect this excess mortality. Indeed, while patients with dementia diagnoses were more likely to present with a more advanced stage of cancer at diagnosis, adjusting for differences in stage at diagnosis had a relatively small effect on deaths in patients with dementia who had breast or colon cancer, and no effect in those with prostate cancer.

These findings do not directly answer the question of whether to screen for cancer in patients with dementia diagnoses, but they do suggest that the potential benefit of such screening is considerably less in patients with dementia diagnoses than in older patients without dementia. This finding is analogous to the results of Satariano and Ragland, ${ }^{18}$ who found a change in the relationship of stage at diagnosis and survival in women with multiple serious comorbidities. In older women with breast cancer and severe comorbidities, early stage at diagnosis conferred no survival advantage. ${ }^{18}$ In view of the limited survival benefits from early cancer diagnosis in older patients with diagnoses of dementia, some authors have recommended informing patients and their caregivers that cancer screening tests such as mammography might pose more harms than benefits in those with dementia diag-noses. ${ }^{19}$ Indeed, the previously reported findings of lower use of invasive diagnostic and staging procedures in patients with cancer who have dementia diagnoses may reflect good clinical judgment on the part of caregivers and clinicians. ${ }^{4,5}$ 
Our study has some limitations, including concerns about validity and completeness of claims data identifying cancer care data. Several studies showed good internal and external validity regarding the use of Medicare claims data for outcomes research in breast, colon, and prostate cancer. ${ }^{20-22}$ One issue is the accuracy and completeness of using Medicare claims in identifying patients with dementia. ${ }^{10,23,24}$ The population of patients with dementia differs from the population of patients who have been given a diagnosis of dementia by their physicians. We are studying the latter population. It would be expected to underrepresent individuals with early or milder dementia ${ }^{23}$ and those with poor access to medical care. Taylor et $\mathrm{al}^{10}$ examined sensitivity of using up to 5 years of Medicare claims in 417 patients known to have a clinical diagnosis of dementia. With 3 years of data from the physician supplier and hospital outpatient claims files, investigators were able to correctly identify $87 \%$ of patients with dementia by using $I C D-9-C M$ dementia codes. ${ }^{10}$

Because Medicare claims likely underreport patients with early dementia, ${ }^{23}$ our findings largely reflect the effect of preexisting diagnoses of moderate to severe dementia on survival after a cancer diagnosis. In addition, we did not include mild cognitive impairment (ICD-9$C M$ diagnosis code 331.83) in our definition of patients with a dementia diagnosis. Thus, our results should not be generalized to patients with early dementia or mild cognitive impairment, who have a longer life expectancy than patients with more advanced dementia. ${ }^{25}$ For example, median survival time for patients with early dementia was about 7 years compared with 3.6 years for patients with moderate to severe dementia (Mini Mental State Examination score, $\leq 7) .{ }^{25}$ In addition, the cognitive resources of patients with early dementia are such that they can still participate in decisions related to cancer screening and treatment. For older patients with more advanced dementia, our data suggest that adherence to cancer care guidelines generated from populations with normal cognition may yield limited survival benefits.

Because our analysis is based on the experiences of older patients with cancer enrolled in fee-for-service Medicare, our findings may not be applicable to younger patients or patients enrolled in Medicare health maintenance organizations. Our study has several strengths, including its large sample size, availability of inpatient and outpatient data, information on cause of death, and a wide geographic representation of the United States.

In summary, the data show that older patients with cancer and a dementia diagnosis have a high mortality, which is mostly from noncancer causes. The data also show attenuation of the relationship between cancer stage at diagnosis and survival. This suggests that cancer screening guidelines generated from nondementia populations may not be appropriate in patients with a diagnosis of dementia. The data on survival could guide clinicians, patients, and caregivers in making more informed decisions about cancer screening in the context of preexisting diagnoses of dementia. Our study also raises important clinical, public health, and ethical questions on follow-up care of the increasing number of older cancer survivors. Does comorbid dementia increase the risk of toxic effects from cancer treatment? What is the appropriate level of care for cancer in the context of preexisting moderate or severe dementia? Are different models of care more appropriate for these patients? ${ }^{26}$ These are complex questions that will require additional studies, including an examination of how dementia affects chemotherapy-related toxic effects, hospitalizations, post-treatment surveillance, cancer recurrence, and hospice use. Such studies may help in development of cancer screening guidelines that consider quality of life and life expectancy of patients living with dementia. ${ }^{24,25}$ 


\section{Acknowledgments}

Funding/Support: Dr Goodwin was supported by grants P50 CA105631 and R01CA104949 from the National Cancer Institute. Dr Raji was supported in part by Public Health Service grant P30-AG-024832-01.

\section{References}

1. Evans DA, Funkenstein HH, Albert MS, et al. Prevalence of Alzheimer's disease in a community population of older persons: higher than previously reported. JAMA. 1989; 262(18):2551-2556. [PubMed: 2810583]

2. Hebert LE, Becket LA, Scherr PA, Evans DA. Annual incidence of Alzheimer disease in the United States projected to the years 2000 through 2050. Alzheimer Dis Assoc Disord. 2001; 15(4):169173. [PubMed: 11723367]

3. Jemal A, Murray T, Ward E, et al. Cancer statistics, 2005. CA Cancer J Clin. 2005; 55(1):10-30. [PubMed: 15661684]

4. Gupta SK, Lamont EB. Patterns of presentation, diagnosis, and treatment in older patients with colon cancer and comorbid dementia. J Am Geriatr Soc. 2004; 52(10):1681-1687. [PubMed: 15450045]

5. Gorin SS, Heck JE, Albert S, Hershman D. Treatment for breast cancer in patients with Alzheimer's disease. J Am Geriatr Soc. 2005; 53(11):1897-1904. [PubMed: 16274370]

6. Goodwin JS, Samet JM, Hunt WC. Determinants of survival in older cancer patients. J Natl Cancer Inst. 1996; 88(15):1031-1038. [PubMed: 8683633]

7. Kukull WA, Brenner DE, Speck CE, et al. Causes of death associated with Alzheimer disease: variation by level of cognitive impairment before death. J Am Geriatr Soc. 1994; 42(7):723-726. [PubMed: 8014346]

8. Warren JL, Klabunde CN, Schrag D, Bach PB, Riley GF. Overview of the SEER-Medicare data: content, research applications, and generalizability to the United States elderly population. Med Care. 2002; 40((8)(suppl)):IV-3-IV-18.

9. Potosky AL, Riley GF, Lubitz JD, Mentnech RM, Kessler LG. Potential for cancer related health services research using a linked Medicare-tumor registry database. Med Care. 1993; 31(8):732-748. [PubMed: 8336512]

10. Taylor DH, Fillenbaum GG, Ezell ME. The accuracy of Medicare claims data in identifying Alzheimer's disease. J Clin Epidemiol. 2002; 55(9):929-937. [PubMed: 12393082]

11. Klabunde CN, Potosky AL, Legler JM, et al. Development of a comorbidity index using physician claims data. J Clin Epidemiol. 2000; 53(12):1258-1267. [PubMed: 11146273]

12. Preacher KJ, Hayes AF. SPSS and SAS procedures for estimating indirect effects in simple mediation models. Behav Res Methods Instrum Comput. 2004; 36(4):717-731. [PubMed: $15641418]$

13. Goodwin JS, Samet JM, Key CR. Stage at diagnosis of cancer varies with the age of the patient. J Am Geriatr Soc. 1986; 34(1):20-26. [PubMed: 3941240]

14. Albertsen PC, Hanley JA, Fine J. 20-year outcomes following conservative management of clinically localized prostate cancer. JAMA. 2005; 293(17):2095-2101. [PubMed: 15870412]

15. Boyd CM, Darer J, Boult C, Fried LP, Boult L, Wu AW. Clinical practice guidelines and quality of care for older patients with multiple comorbid diseases: implications for pay for performance. JAMA. 2005; 294(6):716-724. [PubMed: 16091574]

16. Tinetti ME, Bogardus ST, Agostini JV. Potential pitfalls of disease-specific guidelines for patients with multiple conditions. N Engl J Med. 2004; 351(27):2870-2874. [PubMed: 15625341]

17. Gross CP, McAvay GJ, Krumholz HM, Paltiel AD, Bhasin D, Tinetti ME. The effect of age and chronic illness on life expectancy after a diagnosis of colorectal cancer: implications for screening. Ann Intern Med. 2006; 145(9):646-653. [PubMed: 17088577]

18. Satariano WA, Ragland DR. The effect of comorbidity on 3-year survival of women with primary breast cancer. Ann Intern Med. 1994; 120(2):104-110. [PubMed: 8256968]

19. Raik BL, Miller FG, Fins JJ. Screening and cognitive impairment: ethics of forgoing mammography in older women. J Am Geriatr Soc. 2004; 52(3):440-444. [PubMed: 14962162] 
20. Freeman JL, Klabunde CN, Schussler N, Warren JL, Virnig BA, Cooper GS. Measuring breast, colorectal and prostate cancer screening with Medicare claims data. Med Care. 2002; 40((8) (suppl)):IV-36-IV-42.

21. Lamont EB, Lauderdale DS, Schilsky RL, Christakis NA. Construct validity of Medicare chemotherapy claims: the case of 5FU. Med Care. 2002; 40(3):201-211. [PubMed: 11880793]

22. Warren JL, Harlan LC, Fahey A, et al. Utility of the SEER-Medicare data to identify chemotherapy use. Med Care. 2002; 40((8)(suppl)):IV-55-IV-61.

23. Newcomer R, Clay T. Misclassification and selection bias when identifying Alzheimer's disease solely from Medicare claims records. J Am Geriatr Soc. 1999; 47(2):215-219. [PubMed: 9988293]

24. Taylor DH Jr, Sloan FA, Doraiswamy PM. Marked increase in Alzheimer's disease identified in Medicare claims records between 1991 and 1999. J Gerontol A Biol Sci Med Sci. 2004; 59(7): 762-766. [PubMed: 15304542]

25. Larson EB, Shadlen MF, Wang L, et al. Survival after initial diagnosis of Alzheimer disease. Ann Intern Med. 2004; 140(7):501-509. [PubMed: 15068977]

26. Goodwin JS, Freeman JL, Satish S, Anderson E, Nattinger A. Nurse case managers improve the care of older women with breast cancer. J Am Geriatr Soc. 2003; 51(9):1252-1259. [PubMed: 12919237] 

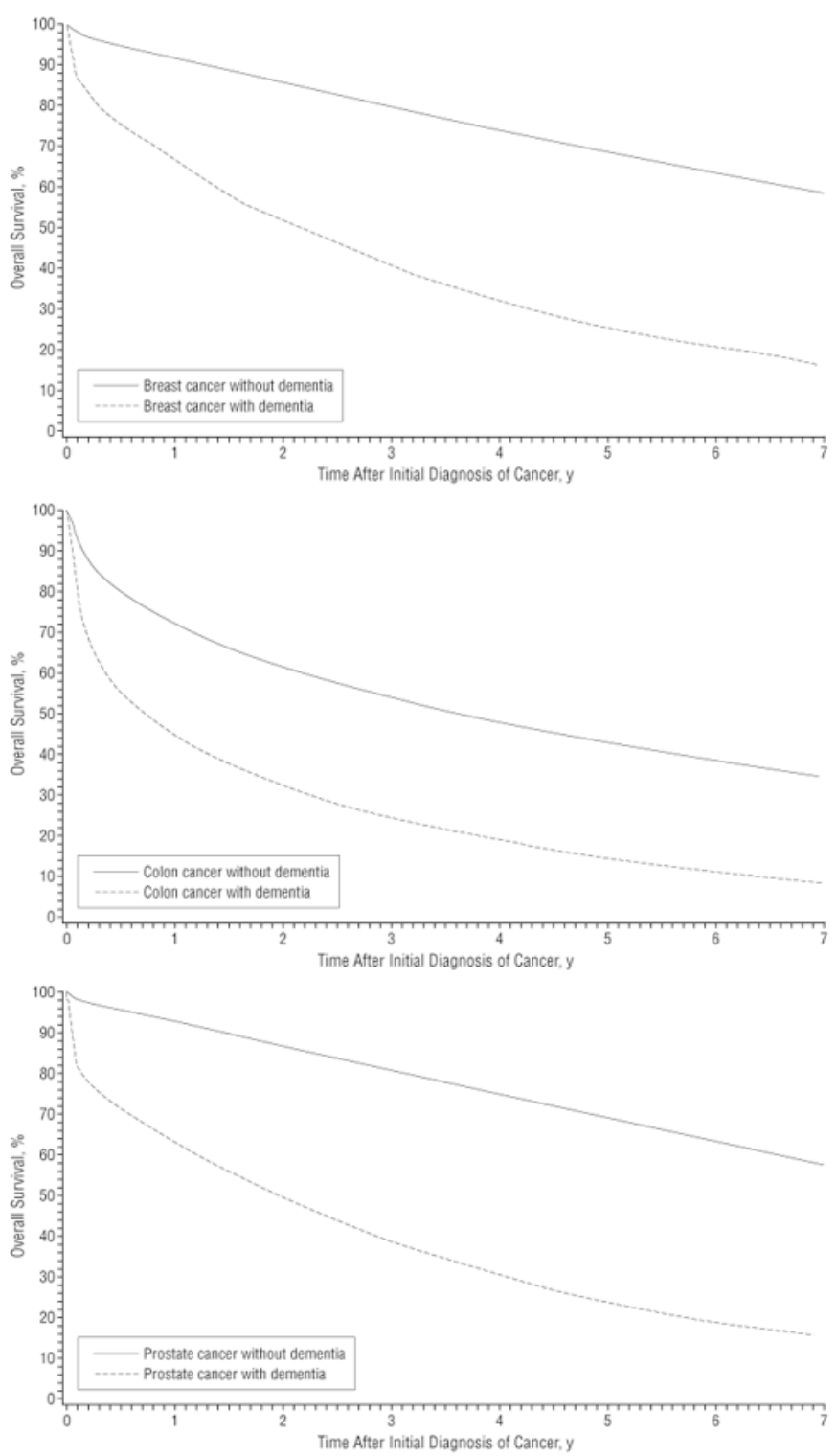

Figure.

Kaplan-Meier survival curves for older patients with breast, colon, and prostate cancer, stratified by whether they carried a preexisting diagnosis of dementia. The group with dementia included Medicare beneficiaries who received a diagnosis of dementia between January 1991 and December 1999, within the 3 years before their cancer diagnosis.

International Classification of Diseases, Ninth Revision, Clinical Modification codes at any visit and in any position from inpatient, hospital outpatient, and physician claims were used to identify patients with a precancer diagnosis of dementia. 
Table 1

\section{Characteristics of Older Patients With Cancer by Dementia Status}

\begin{tabular}{|c|c|c|}
\hline Demographics & No. of Subjects & No. (\%) With Dementia ${ }^{a}$ \\
\hline Entire sample & 106061 & $7453(7.0)$ \\
\hline \multicolumn{3}{|l|}{ Age, y } \\
\hline $68-74$ & 43890 & $1025(2.3)$ \\
\hline $75-84$ & 46925 & $3416(7.3)$ \\
\hline 285 & 15246 & $3021(19.8)$ \\
\hline \multicolumn{3}{|l|}{ Ethnicity } \\
\hline White & 88381 & $5996(6.8)$ \\
\hline Black & 8575 & $941(11.0)$ \\
\hline Hispanic & 3778 & $252(6.7)$ \\
\hline Other & 5327 & $264(5.0)$ \\
\hline \multicolumn{3}{|l|}{ Sex } \\
\hline Female & 47622 & $4138(8.7)$ \\
\hline Male & 58439 & $3315(5.7)$ \\
\hline \multicolumn{3}{|l|}{ Married status } \\
\hline Married & 56484 & $2424(4.3)$ \\
\hline Not married & 49577 & $5029(10.1)$ \\
\hline \multicolumn{3}{|c|}{ Census tract education, $\%$ of adults with $<12$ y of education $b$} \\
\hline$<10$ & 22551 & $1208(5.4)$ \\
\hline 10 to 19 & 35147 & $2188(6.2)$ \\
\hline 20 to 29 & 26088 & $1794(6.9)$ \\
\hline 230 & 17454 & $1429(8.2)$ \\
\hline \multicolumn{3}{|c|}{ Census tract poverty, $\%$ living below poverty line $b$} \\
\hline$<3$ & 18812 & $1049(5.6)$ \\
\hline 3 to 6 & 32064 & $1978(6.2)$ \\
\hline 7 to 13 & 28822 & $1820(6.3)$ \\
\hline$\geq 14$ & 21542 & $1772(8.2)$ \\
\hline \multicolumn{3}{|l|}{ Type of cancer } \\
\hline Prostate & 47235 & $2393(5.1)$ \\
\hline Colon & 26891 & $2691(10.0)$ \\
\hline Breast & 31935 & $2369(7.4)$ \\
\hline \multicolumn{3}{|c|}{ Year of diagnosis } \\
\hline 1994 & 19088 & $1092(5.7)$ \\
\hline 1995 & 18125 & $1104(6.1)$ \\
\hline 1996 & 17419 & $1152(6.6)$ \\
\hline 1997 & 17795 & $1311(7.4)$ \\
\hline 1998 & 16895 & $1391(8.2)$ \\
\hline 1999 & 16739 & $1403(8.4)$ \\
\hline \multicolumn{3}{|c|}{ No. of comorbid conditions, not including dementia } \\
\hline 0 & 73425 & $3807(5.2)$ \\
\hline
\end{tabular}




\begin{tabular}{crr}
\hline Demographics & No. of Subjects & No. (\%) With Dementia ${ }^{a}$ \\
\hline 1 & 21262 & $1932(9.1)$ \\
2 & 7289 & $993(13.6)$ \\
23 & 4085 & $721(17.7)$
\end{tabular}

${ }^{a}$ All differences in prevalences of dementia by demographic characteristics were significant at $P<.001$.

$b_{\text {Of the }} 106061$ subjects with cancer, 4821 subjects had missing data. 
Table 2

Stage at Diagnosis for Patients With Breast, Colon, or Prostate Cancer, Stratified by Whether They Had a Preexisting Diagnosis of Dementia

\begin{tabular}{|c|c|c|c|}
\hline \multirow[b]{2}{*}{ Cancer and Stage } & \multicolumn{2}{|r|}{ No. $(\%)$} & \\
\hline & With Dementia & Without Dementia & \\
\hline Breast $^{a}$ & $n=2369$ & $\mathrm{n}=29566$ & \\
\hline 1 & $663(28.0)$ & $14985(50.7)$ & \\
\hline 2 & $820(34.6)$ & 9212 (31.2) & \\
\hline 3 & $190(8.0)$ & $1702(5.8)$ & \\
\hline 4 & $159(6.7)$ & $1441(4.9)$ & \\
\hline Unknown & $537(22.7)$ & $2226(7.5)$ & \\
\hline Colon $^{a}$ & $\mathrm{n}=2691$ & $\mathrm{n}=24200$ & \\
\hline 1 & $425(15.8)$ & $5152(21.3)$ & \\
\hline 2 & $748(27.8)$ & 7967 (32.9) & \\
\hline 3 & $501(18.6)$ & $5360(22.1)$ & \\
\hline 4 & $412(15.3)$ & $4164(17.2)$ & \\
\hline Unknown & $605(22.5)$ & $1557(6.4)$ & \\
\hline Prostate $^{b}$ & $\mathrm{n}=2393$ & $\mathrm{n}=44842$ & \\
\hline $\mathrm{T} 1$ & $582(24.3)$ & $13040(29.1)$ & \\
\hline $\mathrm{T} 2$ & 797 (33.3) & $22522(50.2)$ & \\
\hline $\mathrm{T} 3$ & $52(2.2)$ & $1618(3.6)$ & \\
\hline $\mathrm{T} 4$ & $295(12.3)$ & $3354(7.5)$ & \\
\hline Unknown & 667 (27.9) & $4308(9.6)$ & \\
\hline \multicolumn{4}{|c|}{$\begin{array}{l}\text { Staging for breast and colon cancer is according to the American Joint Committee on Cancer TNM system. Stage is determined by using } \\
\text { pathological staging data (from surgical assessment and local and regional node evaluation) and clinical staging data (from evaluation of potential } \\
\text { sites for metastasis). All differences in stage at diagnosis for patients with breast, colon, or prostate cancer by dementia status were significant at } \\
P<.001 \text {. }\end{array}$} \\
\hline
\end{tabular}




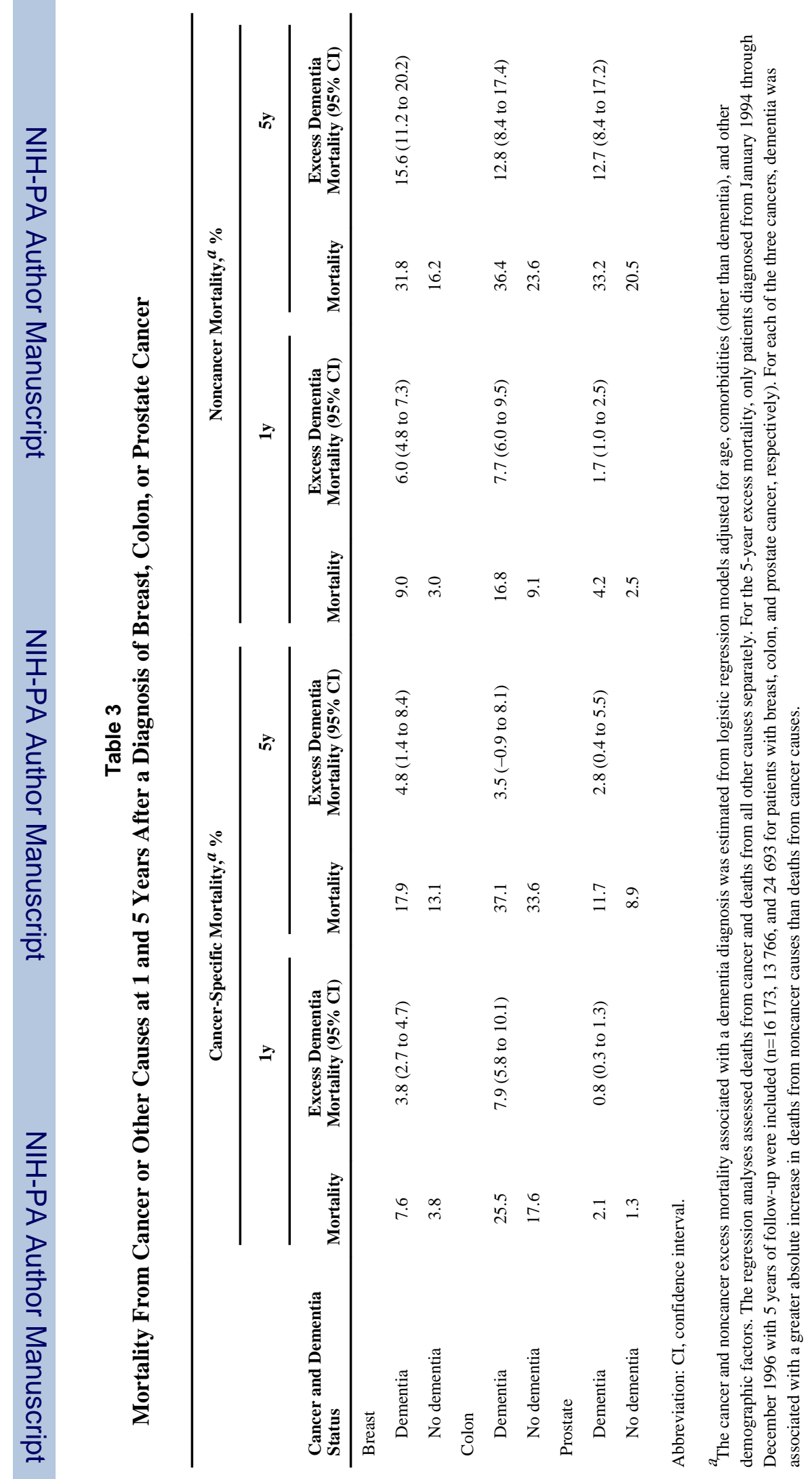

Arch Intern Med. Author manuscript; available in PMC 2013 September 22. 
Table 4

Adjusted HRs of Cancer-Specific and Noncancer Deaths ${ }^{a}$

\begin{tabular}{lccc}
\hline & \multicolumn{3}{c}{ HR $(\mathbf{9 5 \%}$ CI } \\
\cline { 2 - 4 } Type of Cancer & Cancer-Specific Death & Other Causes of Death & Overall Death \\
\hline Colon & & & \\
Model $1 b$ & $1.54(1.44-1.65)$ & $1.89(1.77-2.01)$ & $1.74(1.66-1.82)$ \\
Model $2^{c}$ & $1.44(1.35-1.55)$ & $1.80(1.68-1.92)$ & $1.64^{d}(1.56-1.72)$ \\
Prostate & & & \\
Model $1 b$ & $1.65(1.49-1.83)$ & $1.95(1.83-2.07)$ & $1.95(1.85-2.06)$ \\
Model $2^{c}$ & $1.63(1.47-1.81)$ & $1.93(1.81-2.06)$ & $1.93(1.83-2.04)$ \\
Breast & & & \\
Model $1^{b}$ & $1.77(1.60-1.96)$ & $2.29(2.14-2.45)$ & $2.15(2.03-2.27)$ \\
Model $2^{c}$ & $1.52(1.37-1.68)$ & $2.17(2.03-2.32)$ & $1.96^{d}(1.85-2.07)$
\end{tabular}

Abbreviations: CI, confidence interval; HR, hazard ratio.

${ }^{a}$ Association between a preexisting dementia diagnosis and cancer-specific mortality and overall mortality was estimated from Cox proportional hazard models, with and without additional adjustment for cancer stage, among each type of cancer.

${ }^{b}$ Model 1 adjusts for age, ethnicity, sex (for colon cancer), marital status, Surveillance Epidemiology and End Results region, educational level, income, comorbidity (excluding dementia), year of diagnosis, and grade (estrogen receptor for breast cancer).

${ }^{c}$ Model 2 examines the effect of dementia diagnosis on survival by adding adjustment for cancer stage at diagnosis to the variables included in model 1.

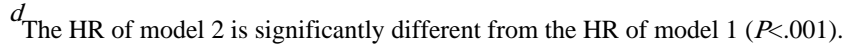




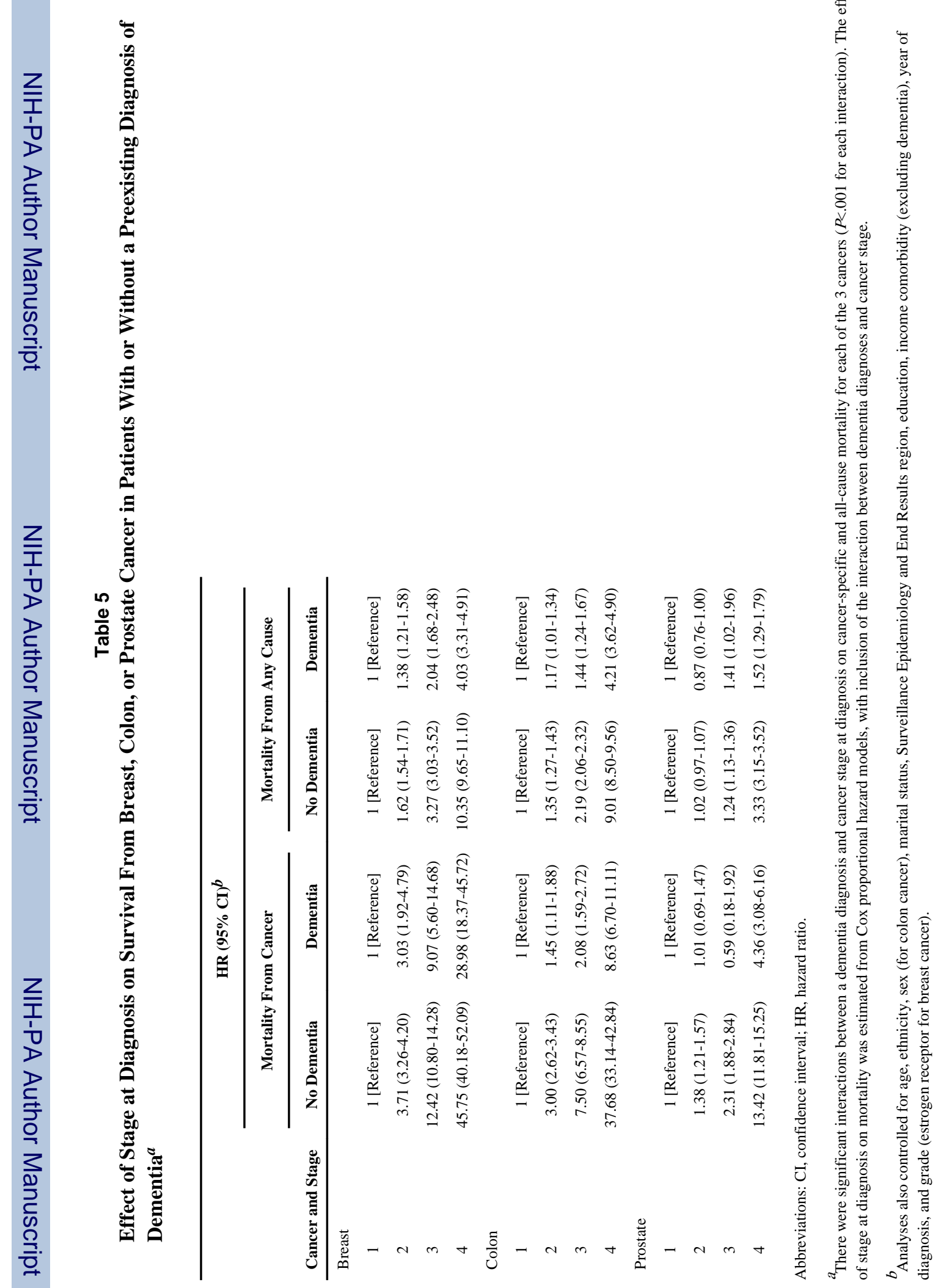

